\title{
EXERCícIOS FÍSICOS E O ENVELHECIMENTO: REVISÃO DE LITERATURE
}

\section{ARTIGO DE REVISÃO}

SILVA, Wender Ambrósio da ${ }^{1}$

SILVA, Verbenia Satelles da ${ }^{2}$

SILVA, Sebastião Lobo da ${ }^{3}$

SANTOS, Givanildo de Oliveira ${ }^{4}$

SILVA, Wender Ambrósio da. Et al. Exercícios físicos e o envelhecimento: Revisão de literatura. Revista Científica Multidisciplinar Núcleo do Conhecimento. Ano 05, Ed. 09, Vol. 03, pp. 119-130. Setembro de 2020. ISSN: 2448-0959, Link de acesso: https://www.nucleodoconhecimento.com.br/educacao-fisica/exercicios-

fisicos

\section{RESUMO}

O conceito de envelhecimento está ligado à um conjunto de fenômenos biológicos, fisiológicos, sociais e psicológicos, que ocorre com todos os seres vivos. Ademais, o envelhecimento é a soma das alterações e adaptações que o organismo sofre com o passar dos anos. O presente estudo tem o objetivo de compreender o processo de envelhecimento do ser humano quanto a suas alterações fisiológicas e biológicas bem como os benefícios das atividades físicas e da musculação. $O$ estudo consiste em uma Revisão da Literatura, realizado por meio da abordagem e inclusão de literatura

\footnotetext{
${ }^{1}$ Bacharel em Educação Física, pela Faculdade Mauá Brasília - DF.

${ }^{2}$ Bacharel em Educação física, pela faculdade de Mauá Brasília - DF.

${ }^{3}$ Doutorando em Educação Física, pela Universidade Católica de Brasília - DF.

${ }^{4}$ Mestre em Tecnologia de Alimentos, pelo Instituto Federal Goiano - Campus Rio Verde - GO.
} 
teórica e empírica, e demais estudos, por meio de abordagens quantitativas e/ou qualitativas, que resultaram na síntese de estudos publicados. Observou-se que praticar atividades físicas regularmente traz consigo uma série de benefícios para a vida, não apenas do idoso, mas para todos aqueles que se dispõe a praticá-la. Os exercícios físicos servem como uma verdadeira ferramenta de manutenção do organismo humano auxiliam, na capacidade cardiorrespiratória, na força, flexibilidade, na resistência muscular, bem como favorece os aspectos psicológicos e emocionais do indivíduo, levando a uma melhor expectativa de vida.

Palavras-chave: Força Muscular, flexibilidade, exercício físico, idoso.

\section{INTRODUÇÃO}

Tem-se observado uma grande alteração na pirâmide etária mundial, sobretudo na brasileira, com o aumento da população idosa e os avanços tecnológicos na área médica, acreditava-se que existiria uma maior qualidade de vida para essa população. No entanto, o envelhecimento da população trouxe consigo um maior número de prevalência de doenças crônicas, principalmente cardiovasculares (ZAGO, 2010).

O envelhecimento é uma característica mundial, que é resultado do crescimento da população idosa. Assim, a qualidade de vida dessa população tem sido objeto de estudos em relação aos seus aspectos gerais, o que eles envolvem e onde interferem. As pesquisas desenvolvidas sobre o processo de envelhecimento e aumento da população idosa estão diretamente ligadas ao âmbito de saúde e envelhecimento, realização regular de exercícios físicos, habilidade funcional e qualidade de vida (CIVINSKI; MONTIBELLER; BRAS, 2011).

Um agente considerável para se obter um envelhecimento saudável é a atividade, podendo ser ela à prática de exercícios físicos individuais ou em grupo. A Organização Mundial da Saúde (OMS) destaca que manter o hábito de realizar exercícios pode prevenir, minimizar e/ou reverter muitos problemas característicos do envelhecimento (HERNANDEZ et al, 2010). 
O assunto tem ganhado cada vez mais visibilidade na mídia. No entanto, é necessário realizar estudos que evidenciem os benefícios dos exercícios frente as doenças e danos do envelhecimento, como um meio de motivar a prática de exercício físico. Ainda é imprescindível o desenvolvimento de pesquisas sobre os efeitos da prática de exercícios físicos e musculação para o envelhecimento humano, destacando as principais características fisiológicas do envelhecimento.

Assim, o estudo tem como objetivo compreender o processo de envelhecimento do ser humano quanto a suas alterações fisiológicas, bem como os benefícios das atividades físicas e da musculação frente a este processo inevitável para o ser humano.

Dessa forma, o envelhecimento é um processo natural que todo homem irá passar. Esse processo pode ocorrer de forma mais brusca ou retardada de acordo com o estilo de vida do indivíduo. A atividade física confere maior força, flexibilidade, autonomia além de influenciar no estado psicológico e de humor da pessoa.

\section{DESENVOLVIMENTO}

\section{METODOLOGIA}

Trata-se de um estudo do tipo bibliográfico, sistematizado e qualitativo. O estudo bibliográfico se baseou em literaturas estruturadas obtidas de livros e artigos científicos provenientes de bibliotecas convencionais e virtuais.

A abordagem qualitativa caracteriza-se pelo emprego de qualificação na coleta e tratamento de informações. O estudo retrospectivo consistiu na obtenção de dados compreendidos no espaço de tempo para análise e discussão dos dados.

Após a definição do tema foram realizadas pesquisas na base de dados virtuais em saúde, e nas seguintes bibliotecas virtuais: Bireme, SCIELO (Scientifc Electroni Library Online), LILACS (Literatura Latino-Americana e do Caribe em Ciências da Saúde); Além de dissertações, monografias de graduação, artigos do Ministério da 
Saúde e artigos científicos de diversas bases de dados. Foram utilizados os descritores: Envelhecimento; Atividade Física; Idoso; Força Muscular.

Após o levantamento bibliográfico, foi realizada a leitura exploratória do material encontrado, obtendo-se uma visão global desse material, que fosse de interesse ou não da pesquisa. Em seguida, iniciou-se a leitura seletiva, que permitiu determinar qual material bibliográfico seria de importância para a pesquisa. No critério de inclusão, foram utilizados artigos com temas associados ao da pesquisa através dos descritores que datam de 2010 a 2019, e no critério de exclusão foram utilizados os artigos com temas não associados aos da pesquisa através dos descritores, e que com a data de publicação inferior a 2010.

Os dados foram fichados por unidades temáticas e analisados de forma descritiva, visando descrever as características de determinado fenômeno ou o estabelecimento de relações entre variáveis através da observação sistemática.

\section{ENVELHECIMENTO: FATORES FISIOLÓGICOS}

O envelhecimento consiste em um processo natural inerente de todo ser vivo. $O$ homem ao envelhecer, acaba sofrendo com diversas mudanças, presente nos movimentos, mesmo nos mais cuidadosos e lentos, este processo é possível ser visualizado pela própria pele que assume um aspecto enrugado, bem como ocorre a perda de massa e a redução da força muscular, a memória passa a ser enfraquecida e falhar, e a flexibilidade tende a diminuir, o que ainda resulta na falta de coordenação e equilíbrio (GABRIEL et al, 2013).

O conceito de envelhecimento está ligado à um conjunto de fenômenos biológicos, fisiológicos, sociais e psicológicos, que ocorre com todos os seres vivos. Ademais, o envelhecimento é a soma das alterações e adaptações que o organismo sofre com o passar dos anos (ZAGO, 2010).

A redução da capacidade funcional ao longo da vida ocorre por diferentes fatores como, por exemplo: fatores genéticos, hábitos e estilo de vida e por fim o estado 
psicoemocional. Deste modo, a participação em um programa de exercícios físicos regulares é essencial, e deve ocorrer de forma efetiva visando reduzir, prevenir e tratar os principais declínios funcionais existentes e associados ao envelhecimento (CIVINSKI; MONTIBELLER; BRAZ, 2011).

O processo do envelhecimento passa a ocorrer a partir do momento do nascimento, e passa a se intensificar a partir dos 40 anos, gerando assim a degradação do corpo humano. Afeta a aparência e a condição física do corpo, no entanto, esse processo varia e é tratado diferentemente para indivíduos de uma forma particular. Algumas pessoas aceitam o processo e permitem que ele ocorra naturalmente, outros buscam formas de amenizar os efeitos do tempo, se utilizando de procedimentos e tratamentos estéticos além de mudanças em seus hábitos (SILVA; BRITTO, 2017).

As mudanças fisiológicas características do envelhecimento são leves, incapazes de gerar inaptidão na fase inicial, contudo, com o avanço dos anos, podem causar limitações na prática de atividades básicas. Durante esse observa-se um conjunto de mudanças nos tecidos, órgãos, músculos e nas capacidades funcionais, o que leva a modificar a flexibilidade, a resistência, a força, a energia, a capacidade cardiorrespiratória, a explosão muscular, a agilidade e equilíbrio (ESQUENAZI; SILVA; GUIMARÃES, 2014).

As principais características do envelhecimento são: cabelos esbranquiçados e mais espessos, pele mais seca e com pouca elasticidade, audição pode se tornar mais difícil, visão diminuída, maior sensibilidade à dor, sistema cardiovascular menos eficiente e expansão torácica restrita. Ainda ocorrem outras alterações que estão diretamente ligadas a mobilidade, sendo elas: diminuição da elasticidade de tecidos moles, articulações mais rígidas e com menor capacidade de absorver impactos, menor elasticidade das cartilagens, menor força muscular, ossos podem apresentar osteoporose além das dificuldades com equilíbrio (SILVEIRA et al., 2010).

Quadro 1: Definições de patologias no processo de envelhecimento 
Artrite

\section{Diabetes}

Doenças

Cardíacas

Hipertensão

arterial

Osteoporose
É uma doença inflamatória crônica, sua causa é desconhecida, porém, sua incidência é muito comum, atinge aproximadamente $2 \%$ da população, estima-se uma prevalência de $4,5 \%$ (sendo mais comum em pessoas com idade entre 55 a 75 anos), devido ao crescimento da população idosa, observa-se que os impactos da artrite se tornaram cada vez mais relevantes.

Doença bastante comum, ocasionada pela ação da insulina que altera o metabolismo da glicose no organismo, bem como das gorduras e proteínas o que gera graves consequências. Aparecimento de doenças como arteriosclerose, e ainda com a redução da distensibilidade da aorta e das grandes artérias, de modo que a condução cardíaca acaba por ficar comprometida e a função barorreceptora é reduzida, sendo uma das maiores causas de mortalidade e morbidade, sendo a doença coronariana o motivo de 70 a $80 \%$ de mortes, há ainda a insuficiência cardíaca congestiva, que também está entre as mais comuns durante a internação hospitalar levando a morbidade e mortalidade na população idosa.

A popular "pressão alta" consiste no aumento da força com a qual o coração bombeia o sangue por meio dos vasos, sendo determinada pelo volume de sangue e a resistência para circular no corpo.

A doença esquelética sistêmica é na verdade a redução da massa óssea e deterioração micro arquitetural do tecido ósseo, leva ao aumento da fragilidade óssea e torna o indivíduo suscetível à fratura, tal processo é considerado inevitável no envelhecimento.

FONTE: FRERE et al., (2011). 


\section{SEDENTARISMO NO ENVELHECIMENTO}

Com o passar dos anos os aspectos físicos do homem foram trocados por máquinas e tecnologias, e deste modo a vida moderna tem sido o motivo do aumento de pessoas com vida sedentária. Não se usa mais aquela força e resistência muscular no trabalho como se usava antigamente, a mão-de-obra foi trocada por máquinas que realizam os trabalhos com menor tempo e maior produtividade (GONÇALVES et al, 2014).

Os exercícios físicos estão associados à boa saúde, prevenindo doenças e melhorando dessa forma a qualidade de vida de quem os praticam. As pessoas que têm uma boa aptidão física estão mais aptas a realizarem tarefas do dia-a-dia, com melhor desempenho, sem apresentar sinais de cansaço, além de apresentar maior agilidade, equilíbrio e resistência à força (UENO et al, 2012).

À medida que aumenta a idade, a capacidade física diminui, a inatividade física domina o indivíduo dando lugar ao envelhecimento físico e, com isso também surge outras alterações orgânicas, a maioria delas está relacionada pela diminuição das funções fisiológicas. Assim, com base em uma pesquisa realizada pelo Ministério do Esporte, por meio da qual buscou-se analisar informações sobre práticas esportivas e atividades físicas relativas, foram realizadas 8.902 entrevistas de brasileiros com idade entre 14 e 75 anos, e por meio desta, observou-se que, 45,9\% dos entrevistados são sedentários, e que apenas $28,5 \%$ no momento da pesquisa praticavam algum tipo de atividade física e por fim, observou-se ainda que $25,6 \%$ aproximadamente ainda praticam algum esporte (BRASIL, 2015).

Pessoas na terceira idade e que não realizam exercícios físicos estão mais suscetíveis aos acidentes do dia a dia. Pelo motivo de não apresentarem um bom equilíbrio, não possuir mais força suficiente, e a resistência ainda dificulta a realização de um maior número de movimentos. O sedentarismo ainda pode levar à doenças crônicas e degenerativas, elevando a incapacidade para prática de exercícios (CIVINSKI; MONTIBELLER; BRAZ, 2011). 
No entanto, aquela clássica imagem de um idoso isolado e dependente está sofrendo modificações com a ideia de "envelhecimento ativo", que tem como objetivo desconstruir ideias impostas por costumes tradicionais e culturais. Sendo que, quando a atividade física é realizada de forma regular, é um dos principais pilares para a manutenção da saúde (FERNANDES, 2014).

Uma grande parcela de idosos já demonstram interesse em manter-se mais saudável, ativo e independente nessa faixa etária, tornando crescente programas e projetos que buscam a promoção da saúde e bem-estar. Esses programas visam incentivar a prática de atividades físicas (esportes, danças, exercícios de força ou atividades recreativas), independente da modalidade, pois todas trazem benefícios para a saúde (FERNANDES, 2014).

\section{BENEFÍCIOS DO EXERCÍCIO FÍSICO PARA A SAÚDE DOS IDOSOS}

Exercícios físicos podem ser definidos com qualquer movimento que envolve um gasto energético de curta (anaeróbico) ou longa (aeróbico) duração, com a finalidade de melhoria das capacidades físicas. Os exercícios aeróbicos são aqueles que se utilizam do oxigênio como fonte de energia para as contrações musculares, sendo de intensidade leve ou moderada. Já os exercícios anaeróbicos são aqueles de intensidade mais elevada, que necessitam de esforço intenso, no entanto de curta duração promovendo a formação de ácido láctico (CARACCIOLO, 2012).

Sendo assim define-se que exercícios aeróbios tendem a desenvolver a resistência muscular e os exercícios anaeróbicos o desenvolvimento da força e potência muscular. Ambos ativam a circulação, fortalece a musculatura, melhora a autoimagem, aumenta a autoestima e produz bem-estar físico.

Diversos estudos evidenciam os benefícios do exercício físico na terceira idade, conforme demonstrado no quadro 1 : 
Quadro 1. Resultados de estudos com intervenção de exercícios físicos.

\begin{tabular}{|c|c|c|c|c|}
\hline $\begin{array}{l}\text { TAMANHO } \\
\text { DA } \\
\text { AMOSTRA }\end{array}$ & $\begin{array}{l}\text { TIPO DE } \\
\text { EXERCÍCIO }\end{array}$ & $\begin{array}{l}\text { INTENSIDADE } \\
\text { DO } \\
\text { EXERCíCIO }\end{array}$ & CONCLUSÃO & REFERÊNCIAS \\
\hline $\begin{array}{l}28 \\
\text { pessoas }\end{array}$ & Lazer e caminhada & $\begin{array}{l}150 \\
\text { min/semana }\end{array}$ & $\begin{array}{l}\text { Atividade física } \\
\text { demonstrou } \\
\text { grande } \\
\text { retardo na } \\
\text { diminuição da } \\
\text { capacidade } \\
\text { funcional. }\end{array}$ & $\begin{array}{l}\text { (CORDEIRO et } \\
\text { al, 2014). }\end{array}$ \\
\hline $\begin{array}{l}20 \\
\text { pessoas }\end{array}$ & $\begin{array}{l}\text { Alongamentos, } \\
\text { treinamento com } \\
\text { peso, circuito, } \\
\text { jogos desportivos, } \\
\text { dança } \\
\text { relaxamento. }\end{array}$ & $\begin{array}{l}3 \text { vezes por } \\
\text { semana com } \\
\text { duração de } 60 \\
\text { minutos. }\end{array}$ & $\begin{array}{l}\text { Os exercícios } \\
\text { físicos } \\
\text { funcionam como } \\
\text { tratamento não } \\
\text { farmacológico e } \\
\text { auxiliam no } \\
\text { equilíbrio, } \\
\text { diminuindo o } \\
\text { risco de queda. }\end{array}$ & $\begin{array}{l}\text { (HERNANDEZ } \\
\text { et al, 2010). }\end{array}$ \\
\hline $\begin{array}{l}40 \\
\text { pessoas }\end{array}$ & Ginástica & $\begin{array}{l}\text { Diário a pelo } \\
\text { menos } \\
\text { meses }\end{array}$ & $\begin{array}{l}\text { Além de ser } \\
\text { benéfico para as } \\
\text { condições } \\
\text { físicas, também } \\
\text { influência } \\
\text { diretamente no } \\
\text { perfil de humor } \\
\text { dos idosos. }\end{array}$ & $\begin{array}{l}\text { (GABRIEL et al, } \\
\text { 2013). }\end{array}$ \\
\hline
\end{tabular}




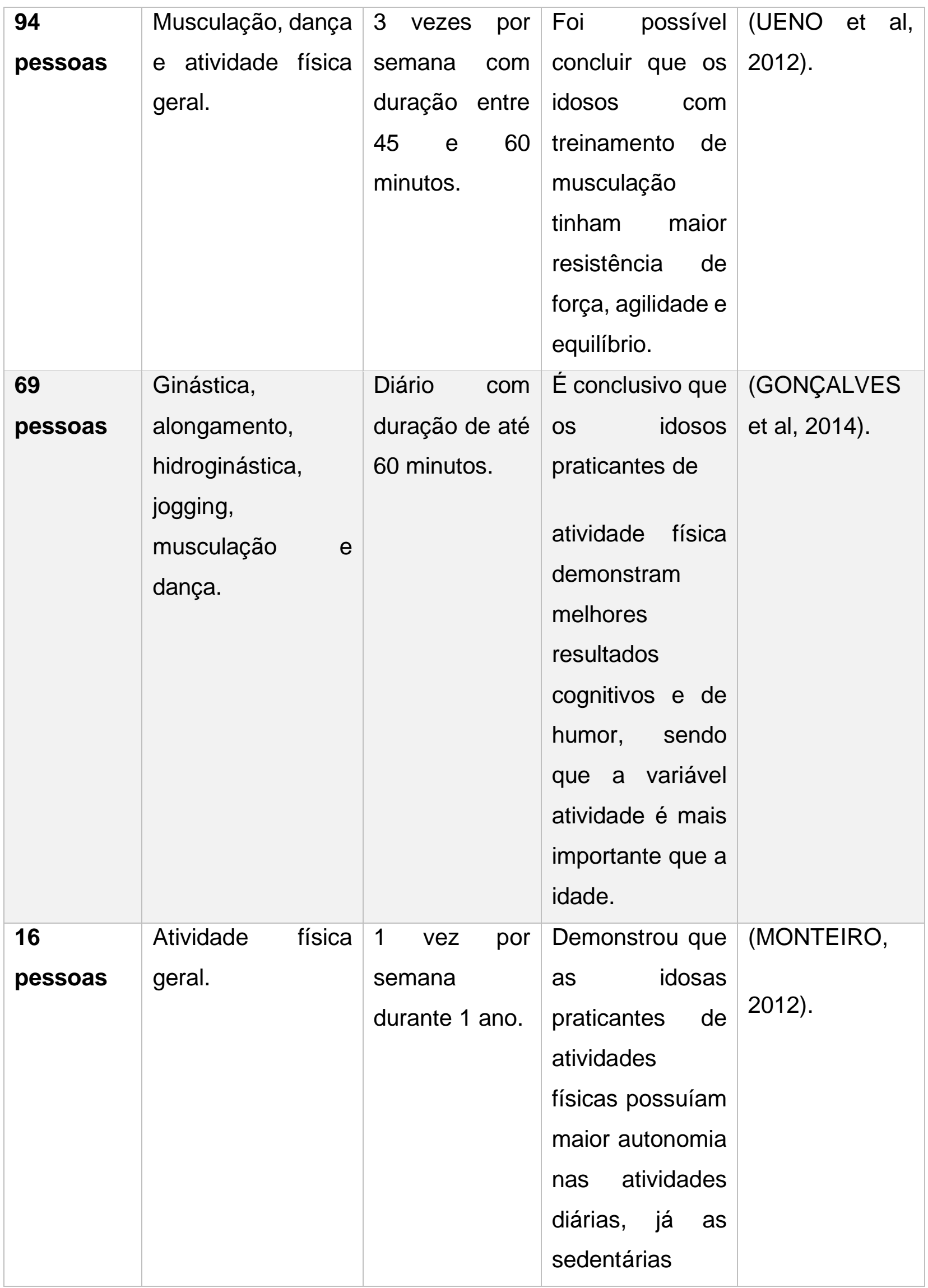




\begin{tabular}{|c|c|c|c|c|}
\hline & & & $\begin{array}{l}\text { apresentavam } \\
\text { dependência por } \\
\text { terceiros. }\end{array}$ & \\
\hline $\begin{array}{l}83 \\
\text { pessoas }\end{array}$ & $\begin{array}{l}\text { Atividade física } \\
\text { geral. }\end{array}$ & $\begin{array}{l}\text { Diária e na } \\
\text { realização de } \\
\text { atividades } \\
\text { cotidianas. }\end{array}$ & $\begin{array}{l}\text { Existe resultado } \\
\text { positivo entre } \\
\text { atividade física e } \\
\text { desempenho } \\
\text { cognitivo em } \\
\text { homem, e nas } \\
\text { mulheres a } \\
\text { relação é entre o } \\
\text { desenvolvimento } \\
\text { aeróbico. }\end{array}$ & $\begin{array}{l}\text { (SOARES et al, } \\
\text { 2013). }\end{array}$ \\
\hline 327 & $\begin{array}{l}\text { Hidroginástica, } \\
\text { natação } \\
\text { ginástica. }\end{array}$ & $\begin{array}{l}2 \text { ou } 3 \text { vezes } \\
\text { por semana } \\
\text { com duração } \\
\text { de } 50 \text { minutos. }\end{array}$ & $\begin{array}{l}\text { Idosos com } \\
\text { osteoporose na } \\
\text { sua maioria } \\
\text { realizam } \\
\text { atividades } \\
\text { aeróbicas, } \\
\text { porém, o estudo } \\
\text { evidencia a } \\
\text { necessidade de } \\
\text { atividades que } \\
\text { visem } \\
\text { fortalecimento } \\
\text { muscular } \\
\text { flexibilidade. }\end{array}$ & $\begin{array}{l}\text { (MAZO et al, } \\
\text { 2013). }\end{array}$ \\
\hline 23 & $\begin{array}{l}\text { Alongamento, } \\
\text { dança, movimento } \\
\text { com alteres e } \\
\text { caminhada. }\end{array}$ & $\begin{array}{l}1 \text { vez por } \\
\text { semana com } \\
\text { duração de até } \\
60 \text { minutos. }\end{array}$ & $\begin{array}{l}\text { A atividade } \\
\text { física } \\
\text { desempenha } \\
\text { papel na }\end{array}$ & $\begin{array}{l}\text { (ALVES et al, } \\
\text { 2013). }\end{array}$ \\
\hline
\end{tabular}




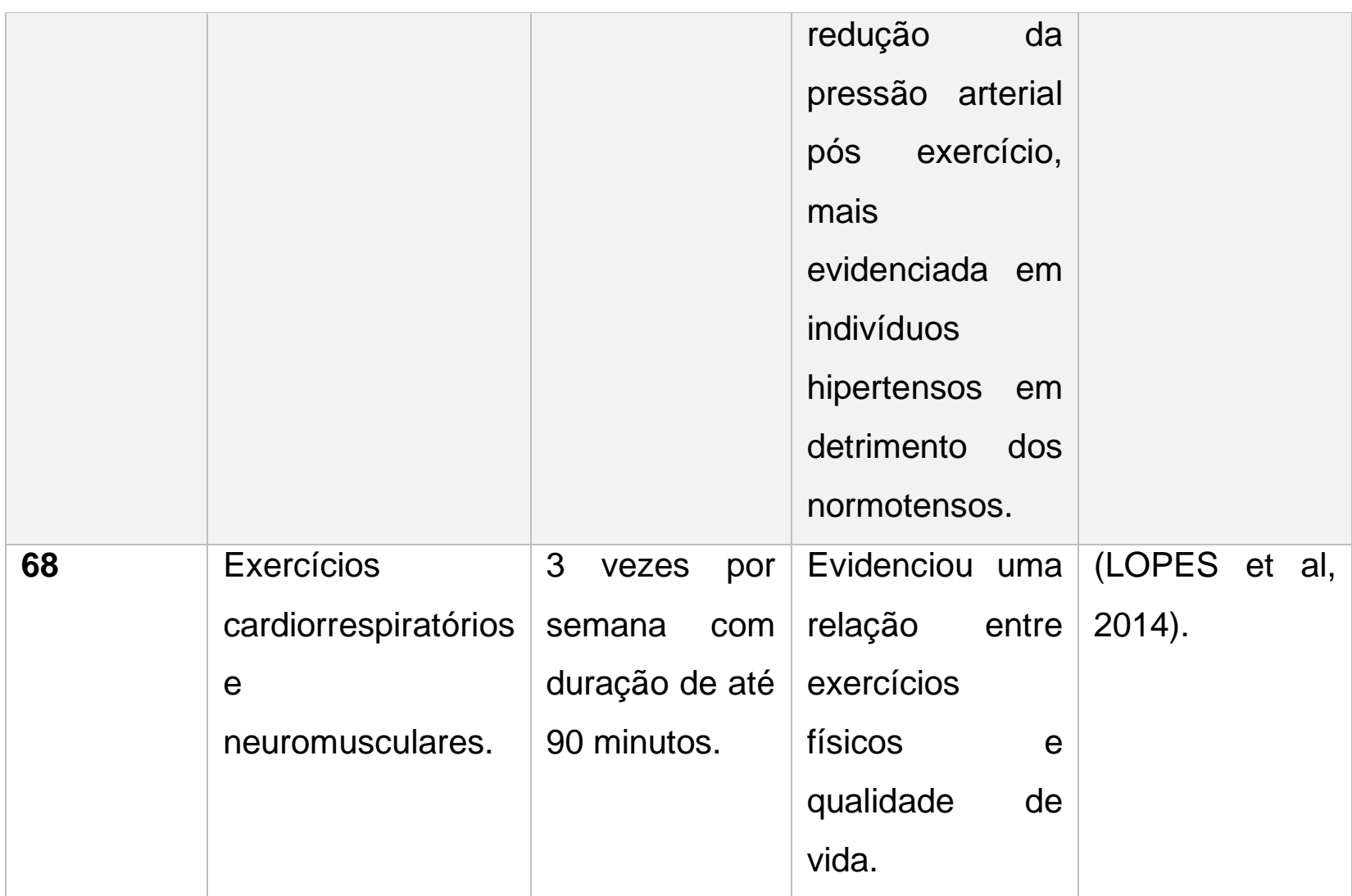

Fonte: autor.

A adoção de práticas saudáveis mantém a autonomia dos idosos, permitindo que realizem suas atividades diárias com mais disposição, reintegra o indivíduo a seus grupos sociais e diminui o risco de depressão. Exercícios podem contribuir para a prorrogação do tempo de vida e permite o progresso do idoso (FERNANDES, 2014).

As alterações fisiológicas que ocorrem no aparelho locomotor do ser humano em decorrência do envelhecimento, como por exemplo, a perda de massa muscular, do equilíbrio corporal, a redução da massa óssea e osteoartrose são as principais causas das limitações às atividades da vida diária do idoso. Assim, observa-se que nos casos de idosos que realizam atividade física de forma periódica, é possível observar melhor independência funcional e qualidade de vida se comparado a outro que seja sedentário (CORDEIRO et al, 2014).

A prática constante de exercícios físicos proporciona o ganho de força e massa muscular, maior flexibilidade nas articulações, o que diminui a incidência de quedas e 
permite que o idoso continue a realização de suas atividades diárias, mesmo aqueles portadores de demência como Alzheimer (HERNANDEZ; et al, 2010).

Exercícios físicos de forma geral atuam no aumento da independência e autonomia na realização das atividades cotidianas. No entanto, observa-se que os efeitos, muitas vezes, dependem do tipo de exercício realizado, como, por exemplo, no caso dos exercícios de flexibilidade, há melhora da marcha e redução de quedas; no caso dos exercícios de força, observou-se que estes atuam na estabilidade postural e redução das quedas; e, no caso dos exercícios aeróbios há o aumento da resistência aeróbica e ajudam no controle das doenças crônicas (SOUZA; FARAH, 2016).

A manutenção da capacidade funcional dos idosos é um dos fatores que mais tem contribuído para uma melhor qualidade de vida desta fatia população, assim, evidencia-se que a prática de atividades físicas é uma forma relevante de alcançar esse objetivo, e por isso, deve ser estimulada ao longo da vida (MACIEL, 2010).

\section{CONSIDERAÇÕES FINAIS}

É evidente as grandes alterações fisiológicas que ocorrem durante o processo de envelhecimento. Mudanças essas que ocorrem de forma gradual e quase de forma imperceptível, mas que com o passar dos anos começam a apresentar sinais que refletem na aparência, mobilidade e na fisiologia do idoso. Essas alterações na fisiologia são os principais responsáveis por construir uma visão de idosos dependentes e isolados da sociedade.

Os exercícios físicos são grandes aliados no retardo do processo de envelhecimento, ajudando na melhor qualidade de vida, aumento da mobilidade, força muscular e aliada no tratamento de doenças crônicas e degenerativas comuns da terceira idade. Os estudos evidenciaram uma diferença significativa entre idosos sedentários e aqueles adeptos de atividade física regular.

Demonstraram ainda que idosos ativos ainda possuem uma mobilidade superior até quando comparados a jovens sedentários. Sendo assim, cabe ao profissional de 
educação física, atuar com compromisso com a prática de ações regulares que proporcionem atividades para melhora da qualidade de vida, independência e para as atividades diárias do adulto em fase de envelhecimento, visando principalmente o equilíbrio do bem-estar físico, emocional e o social para que prolongue a vida com qualidade, bem como a força muscular e das articulações.

\section{REFERÊNCIAS}

ALVES, C. B; et al. Pressão arterial de idosos que praticam atividade física em um grupo de envelhecimento saudável. Arquivo de Ciência do Esporte, vol.1, n.1, p. 7680, 2013.

BRASIL. MINISTÉRIO DO ESPORTE. A prática de esporte no Brasil. Esplanada dos Ministérios, Brasília-DF, 2015.

CARACCIOLO, M. Queima Calorias. Revista Saúde \&Lar, Publicadora Servir. Sabugo, n. 773, 2012.

CIVINSKI, C; MONTIBELLER, A; BRAZ, A. L. O. A importância do exercício físico no envelhecimento. Revista da Unifebe (online), vol.9, p.163-175, 2011.

CORDEIRO, J; et al. Efeitos da atividade física na memória declarativa, capacidade funcional e qualidade de vida em idosos. Revista Brasileira de Geriatria e Gerontologia, Rio de Janeiro, vol. 17, n.3, p.541-552, 2014.

ESQUENAZI, D; SILVA, S. R. B; GUIMARÃES, M. A. M. Aspectos fisiopatológicos do envelhecimento humano e quedas em idosos. Revista HUPE, Rio de Janeiro, vol.13, n.2, p.11-20, 2014.

FERNANDES, B. L. V. Atividade física no processo de envelhecimento. Revista Portal de Divulgação, ano 4, n.40, p. 43-48, 2014. 
GABRRIEL, C. B; et al. Efeitos agudos da atividade física sobre o estado de humor de indivíduos na 3ai idade. Revista Saúde Física \& Mental, UNIABEU, vol.2, n.1, p. 11-21, 2013.

GONÇALVES, A. K; et al. Qualidade de vida e sintomas depressivos em idosos de três faixas etárias praticantes de atividade física. Revista Kairós de Gerontologia, São Paulo, vol.17, n.3, p. 79-94, 2014.

HERNANDEZ, S. S. S; et al. Efeitos de um programa de atividade física nas funções cognitivas, equilíbrio e risco de quedas em idosos com demência de Alzheimer. Revista Brasileira de Fisioterapia, São Carlos, vol.14, n.1, p.68-74, 2010.

LOPES, L. C; et al. Efeitos da atividade física na qualidade de vida de mulheres com sobrepeso e obesidade pós menopausa. Ciência, Cuidado e Saúde, vol.13, n.2, p. 439-446, 2014.

MACIEL, M.G. Atividade física e funcionalidade do idoso. Motriz, Rio Claro, v.16, n.4, p.1024-1032, 2010.

MAZO, G. Z; et al. Associação entre osteoporose e aptidão física de idosos praticantes de exercícios físicos. SAÚDE, Santa Maria, vol. 39, n.2, p. 131-140, 2013.

MONTEIRO, R. Atividade física, aptidão física e grau de dependência nas ABVDS e AIVDS. Journal of Aging and Inovation, vol.1, n.5, p. 45-54, 2012.

PEDRINELLI, A; GARCEZ-LEME, L. E; NOBRE, R. S. A. O efeito da atividade física no aparelho locomotor do idoso. Revista Brasileira de Ortopedia. São Paulo, vol. 44, n. 2 , p. $96-101,2009$.

SILVA, O. M; BRITTO, J. Q. A. O avanço da estética no processo de envelhecimento: uma revisão de literatura. Id Online. Revista Multidisciplinar e de Psicologia, vol.11, n.38, p. 424-440, 2017.

SILVEIRA, M. M; et al. Envelhecimento humano e as alterações na postura corporal do idoso. Revista Brasileira de Ciências da Saúde, ano 8, n.26, p. 56-58, 2010. 
SOARES, R. M. Associação entre atividade física, aptidão física e desempenho cognitivo em idosos. Motricidade, vol.9, n.2, p. 85-94, 2013.

SOUZA, R.C.C; FARAH, B.Q. Efeitos dos exercícios físicos nas atividades da vida diária em idosos: uma revisão bibliográfica. Disponível em:<http://repositorio.asces.edu.br/jspui/bitstream/123456789/540/1/TCC\%20\%20R ENATO\%20C\%C3\%89SAR_FINAL.pdf>. Acesso em 5 out. 2019.

UENO, D. T; et al. Efeitos de três modalidades da atividade física na capacidade funcional de idosos. Revista Brasileira de Educação Física e Esportes, São Paulo, vol.26, n.2, p. 273-281, 2012.

ZAGO, A. S. Exercício físico e o processo saúde-doença no envelhecimento. Revista Brasileira de Geriatria e Gerontologia, Rio de Janeiro, vol.13, n.1, p.153-158, 2010.

Enviado: Agosto, 2020.

Aprovado: Setembro, 2020. 